\title{
Reclassification of asymptomatic beta cell autoimmunity: a critical perspective
}

\author{
Mikael Knip $^{1,2,3,4} \cdot$ Jenni Selvenius $^{3} \cdot$ Heli Siljander $^{1,2} \cdot$ Riitta Veijola $^{5}$
}

Received: 17 August 2016/Accepted: 28 September 2016 /Published online: 27 October 2016

(C) Springer-Verlag Berlin Heidelberg 2016

\begin{abstract}
Type 1 diabetes is an immune-mediated disease leading to almost total beta cell destruction and permanent exogenous insulin dependency. The appearance of clinical symptoms is preceded by an asymptomatic preclinical period, the duration of which is highly individual. The emergence of diabetes-associated autoantibodies into the peripheral circulation is the first detectable sign of beta cell autoimmunity. If type 1 diabetes is diagnosed in childhood the preclinical period lasts for an average of 2.5-3 years, but clinical symptoms may in some cases appear within a few months or be delayed for more than 20 years. In this issue of Diabetologia, Bonifacio and colleagues (doi:10.1007/s00125-016-4144-8) suggest that asymptomatic beta cell autoimmunity should be considered as a pathological and diagnostic entity. Although such a strategy may have some positive consequences, it might also have serious drawbacks. To label an asymptomatic child that may have 10-20 years of a healthy life ahead of him/her as a patient will most likely affect both the life of the family and the child. Therefore, we think that one should not adapt the new
\end{abstract}

Mikael Knip

mikael.knip@helsinki.fi

1 Children's Hospital, University of Helsinki and Helsinki University Hospital, P.O. Box 22 (Stenbäckinkatu 11), 00014 Helsinki, Finland

2 Research Programs Unit, Diabetes and Obesity, University of Helsinki, Helsinki, Finland

3 Tampere Center for Child Health Research, Tampere University Hospital, Tampere, Finland

4 Folkhälsan Research Center, Helsinki, Finland

5 Department of Pediatrics, PEDEGO Research Unit (Research Unit for Pediatrics, Dermatology, Clinical Genetics, Obstetrics and Gynecology), Medical Research Center, Oulu University Hospital and University of Oulu, Oulu, Finland diagnosis before the psychological consequences of such a strategy have been assessed. Instead, since metabolic abnormalities precede the appearance of clinical symptoms of type 1 diabetes, analysis of a combination of immunological and metabolic markers will provide better insight into the likelihood of progression to clinical disease, with a shorter 'sickness' period.

Keywords Clinical science $\cdot$ Human $\cdot$ Prediction and prevention
Abbreviations
ABCD Autoimmune Beta Cell Disorder
DIPP Type 1 Diabetes Prediction and Prevention
IFG Impaired fasting glucose
IGT Impaired glucose tolerance

\section{Is it justified to label an asymptomatic autoantibody-positive child as a patient?}

The position paper by Bonifacio et al argues that asymptomatic beta cell autoimmunity should be perceived as a disorder, representing a true diagnosis reflective of a medical condition [1]. This is a controversial proposal that needs broad discussion and thorough consideration among researchers, clinicians, healthcare authorities and affected families. The most critical issue, in our view, is whether it is justified to label an asymptomatic child with a normal glucose tolerance as a patient with a disease. We are aware that the overwhelming majority of young children who test persistently positive for two or more beta cell autoantibodies will eventually present with clinical type 1 diabetes [2]. However, a proportion of such children will experience one or more inverse seroconversions [3]. According to The Environmental Determinants of 
Diabetes in the Young (TEDDY) study, that proportion was only $1.4 \%$ when including genetically predisposed children who tested persistently positive (in at least two sequential samples) for multiple biochemical autoantibodies [3], but in the Type 1 Diabetes Prediction and Prevention (DIPP ) study 9.3\% of children with HLA-conferred disease susceptibility who had two or more autoantibodies on at least two occasions experienced an inverse seroconversion (J. Selvenius, R. Veijola, H. Siljander, M. Knip, unpublished data). Such children have a clearly reduced risk of progression to clinical type 1 diabetes (Fig. 1). This suggests that a nontrivial proportion of children testing positive for multiple autoantibodies with low progression risk would be labelled as being sick. Moreover, the suggested abbreviation of autoimmune beta cell disorder (ABCD) is already used for other conditions, such as peroxisome dysfunction disorders [4].

It is not possible to predict when type 1 diabetes will manifest based on the autoantibody status, and, as previously mentioned, the interval from seroconversion to the appearance of clinical symptoms may last for more than 20 years [5]. Accordingly an asymptomatic autoantibody-positive individual may remain normoglycaemic and healthy, whilst carrying the patient label for several decades. What will the psychological consequences be? How will the family react to this and how will the children themselves handle the situation? More information and experience is needed regarding these issues, including recommendations for clinical follow-up and psychological support for these families.

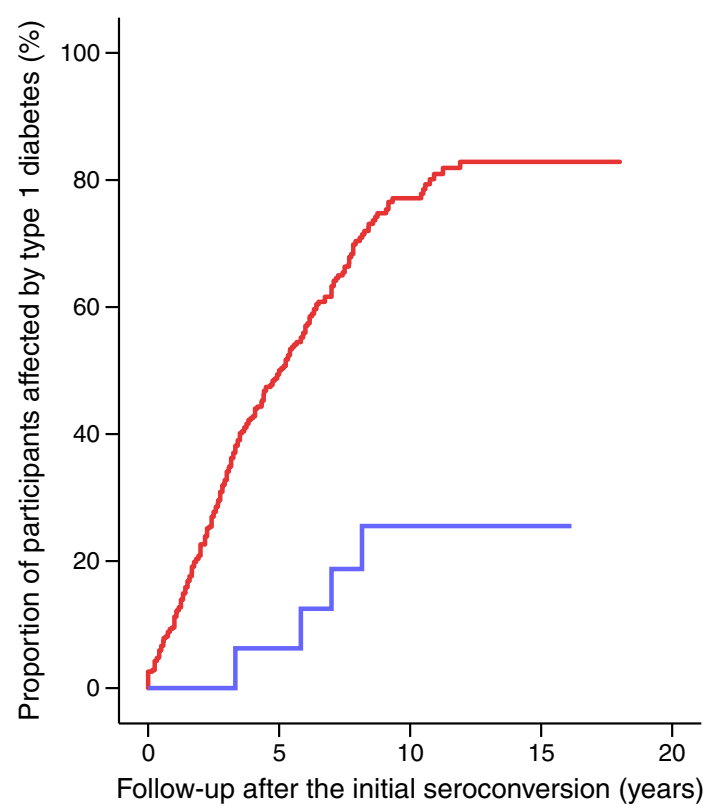

Fig. 1 Progression to clinical type 1 diabetes among 55 children who tested positive for at least two autoantibodies and experienced an inverse seroconversion after persistent multipositivity (blue line) compared with the 537 remaining participants who were positive for multiple autoantibodies during the whole follow-up time (red line). $p<0.001$, Kaplan-Meier survival analysis

\section{Pros and cons of the autoimmune beta cell disorder concept}

We think that the proposal by Bonifacio et al is premature, since there are still many questions and open issues around the cause and disease process in type 1 diabetes. We are afraid that their proposal would result in a scenario where asymptomatic autoantibody-positive children with normal glucose tolerance are labelled as patients, although they may have a healthy life for many years ahead of diagnosis. We do not know the consequences of the patient label on the child and his/her family. At least, the consequences of the patient label should be studied before going forward. Our long-term experience from large prospective clinical studies suggests that families with a child persistently positive for multiple beta cell autoantibodies can be informed about the significance of this finding by using various stages of type 1 diabetes, which have been published recently [6]. Stage 1 indicates a presymptomatic/ preclinical stage with beta cell autoimmunity; stage 2, beta cell autoimmunity and dysglycaemia; and stage 3, symptomatic type 1 diabetes. The same ICD-10 code (endocrine, nutritional and metabolic diseases (E)10: Type 1 diabetes) can be used for all of these stages; thus, a new disease code would be unnecessary. Instead of a new diagnosis we would, therefore, prefer the well-accepted concept of subclinical disease states for type 1 diabetes. This is already widely used for conditions such as infections and malignancies. This concept would also take into account the fact that progression time to clinical disease varies between individuals and may last for several years or even decades. In addition, there would be the possibility that no progression to clinical disease may occur, which may be relevant for those with multiple islet autoantibodies but no signs of dysglycaemia.

Bonifacio et al state that making the diagnosis in the asymptomatic stage, prior to clinical manifestation, has the potential to reduce the threat of ketoacidosis, alleviate psychological burden, allow for earlier initiation of experimental treatments that may preserve insulin sufficiency and reduce healthcare costs. A closer analysis of these arguments shows that some are well justified, whereas others are not. There are several reports showing that participation in prospective studies with sequential autoantibody testing substantially reduces the frequency of diabetic ketoacidosis at diagnosis in those who present with clinical diabetes [7, 8]. As far as we are aware, there are no studies suggesting that an early diagnosis of type 1 diabetes reduces the psychological burden related to the clinical disease. Increased anxiety and worry are common reactions among parents when learning that their child is at increased risk for type 1 diabetes [9]. In most cases these reactions dissipate with time but some parents may be particularly vulnerable to prolonged anxiety or depression. In contrast with the early detection of other diseases, such as rheumatoid arthritis, where there are drugs that effectively remove 
the symptoms, although they do not cure the disease [10], it seems ethically questionable to cause such anxiety without any means of relieving it by offering preventive treatment. So far there is no effective and safe experimental treatment capable of preserving insulin secretion to such an extent that a child with diabetes-related autoantibodies remains free of the need for exogenous insulin.

\section{Beta cell autoimmunity vs insulitis}

Regarding type 1 diabetes, there is likely consensus that the disease is an immune-mediated disorder but not necessarily an autoimmune disease. It may, for example, be an infectious disease. Available data from autoantibody-positive organ donors suggest that there is a lack of correlation between the number of detectable autoantibodies in peripheral blood and signs of ongoing disease in the pancreatic islets $[11,12]$. In a Belgian study on 62 autoantibody-positive donors, insulitis was observed in two donors, both having multiple diabetes-associated autoantibodies and a highrisk HLA genotype [11]. Another study, using donors from the Nordic Network for Islet Transplantation, identified 32 autoantibody-positive individuals, nine of whom had multiple autoantibodies, all without any signs of insulitis [12]. We know that the presence of multiple autoantibodies is associated with a substantially higher risk of progression to overt disease than positivity for a single autoantibody. The data available showing a weak association between autoantibody positivity and insulitis in the target organ [12], however, makes it challenging to argue that humoral beta cell autoimmunity would represent a pathological entity or disease.

\section{Combination of autoimmune and metabolic markers}

Given that the proposed new diagnosis of $A B C D$ for asymptomatic individuals with two or more beta cell autoantibodies [1] is associated with a series of limitations and drawbacks, one may prefer staging of asymptomatic or subclinical type 1 diabetes based on both autoimmune and metabolic findings. Based on data from the DIPP study, we have shown that dysglycaemia precedes the clinical diagnosis of type 1 diabetes, with a considerably shorter interval period than that from the time of seroconversion to the appearance of clinical symptoms $[13,14]$. A $10 \%$ rise in the $\mathrm{HbA}_{1 \mathrm{c}}$ levels in samples obtained 3-12 months apart predicted the diagnosis of clinical disease after a median time of 1.1 years from the observed rise in $\mathrm{HbA}_{1 \mathrm{c}}$. Also, where the $\mathrm{HbA}_{1 \mathrm{c}}$ level was $\geq 5.9 \%(41 \mathrm{mmol} / \mathrm{mol})$ in two consecutive samples, the median time to diagnosis was 0.9 years [13]. Furthermore, impaired fasting glucose (IFG) and impaired glucose tolerance (IGT), as defined by
WHO [15], are definite risk factors for type 1 diabetes, as is a random plasma glucose value $\geq 7.8 \mathrm{mmol} / 1$ [14]. The median time to diagnosis after the detection of IFG was 5.2 years, after IGT, 0.7 years and after a random plasma glucose $\geq 7.8 \mathrm{mmol} / 1,1.0$ years. In a retrospective analysis both the OGTT-derived $2 \mathrm{~h}$ plasma glucose concentration and random plasma glucose level started to increase 1.5 years before diagnosis. These observations indicate that a metabolic diagnosis of asymptomatic diabetes reduces the risk of diabetic ketoacidosis as effectively as the proposed $\mathrm{ABCD}$ diagnosis but shortens the 'sickness' period considerably. The limitation of this metabolic diagnosis is a relatively modest sensitivity for clinical diabetes, ranging from only $6 \%$ for IFG to $57 \%$ for a $10 \%$ increase in $\mathrm{HbA}_{1 \mathrm{c}}$ over 3-12 months. New approaches, such as continuous glucose monitoring (CGM), which provides information about glycaemic variability, may be useful tools in future studies and in the assessment of children with persistent beta cell autoimmunity.

Currently there is no effective treatment to reverse or delay the progression of beta cell autoimmunity. If such a treatment becomes available, the scenario would change dramatically and the indication for use of such treatment would be persistent positivity for multiple islet autoantibodies. However, for the time-being, using a combination of autoimmune and metabolic markers, may better serve to assess disease status in asymptomatic individuals than the introduction of the $\mathrm{ABCD}$ concept.

Acknowledgements The authors thank the children and families taking part in the Finnish Type 1 Diabetes Prediction and Prevention (DIPP) study for their feedback on the various aspects of the study.

Funding The authors were supported by the JDRF, Academy of Finland (Centre of Excellence in Molecular Systems Immunology and Physiology Research 2012-2017, Decision No. 250114), Special Research Funds for University Hospitals in Finland; Diabetes Research Foundation, Finland, and Sigrid Jusélius Foundation.

Duality of interest The authors declare that there is no duality of interest associated with this manuscript.

Contribution statement All authors were responsible for drafting the article and revising it critically for important intellectual content. All authors approved the version to be published.

\section{References}

1. Bonifacio E, Mathieu C, Nepom G et al (2016) Rebranding asymptomatic type 1 diabetes: the case for autoimmune beta cell disorder as a pathological and diagnostic entity. Diabetologia doi:10.1007 /s00125-016-4144-8 
2. Ziegler AG, Rewers M, Simell O et al (2013) Seroconversion to multiple islet autoantibodies and risk of progression to diabetes in children. JAMA 309:2473-2479

3. Vehik K, Lynch KF, Schatz DA et al (2016) Reversion of $\beta$-cell autoimmunity changes risk of type 1 diabetes: TEDDY study. Diabetes Care 39:1535-1542

4. Baker A, Carrier DJ, Schaedler T, Waterham HR, van Roermund CW, Theodoulou FL (2015) Peroxisomal ABC transporters: functions and mechanism. Biochem Soc Trans 43:959-965

5. Knip M, Korhonen S, Kulmala P et al (2010) Prediction of type 1 diabetes in the general population. Diabetes Care 33:1206-1212

6. Insel RA, Dunne JL, Atkinson MA et al (2015) Staging presymptomatic type 1 diabetes: a scientific statement of JDRF, the Endocrine Society, and the American Diabetes Association. Diabetes Care 38:1964-1974

7. Elding Larsson H, Vehik K, Bell R et al (2011) Reduced prevalence of diabetes ketoacidosis at diagnosis of type 1 diabetes in young children participating in longitudinal follow-up. Diabetes Care 34: 2347-2352

8. Elding Larsson H, Vehik K, Gesualdo P et al (2014) Children followed in the TEDDY study are diagnosed with type 1 diabetes at an early stage of disease. Pediatr Diabetes 15:118-126
9. Bennett Johnson S (2011) Psychological impact of screening and prediction in type 1 diabetes. Curr Diab Rep 11:454-459

10. Smolen JS, Aletaha D, McInnes IB (2016) Rheumatoid arthritis. Lancet. doi:10.1016/S0140-6736(16)30173-8

11. In't Veld P, Lievens D, De Grijse J et al (2007) Screening for insulitis in adult autoantibody-positive organ donors. Diabetes 56 : 2400-2404

12. Wiberg A, Granstam A, Ingvast $\mathrm{S}$ et al (2015) Characterization of human organ donors testing positive for type 1 diabetes-associated autoantibodies. Clin Exp Immunol 182:278-288

13. Helminen O, Aspholm S, Pokka T et al (2015) HbA1c predicts time to diagnosis of type 1 diabetes in children at risk. Diabetes 64: $1719-1727$

14. Helminen O, Aspholm S, Pokka T et al (2015) Oral glucose tolerance test and random plasma glucose in prediction of type 1 diabetes. Diabetologia 58:1787-1796

15. World Health Organization (2006) Summary of technical report and recommendations. In: WHO, Definition and diagnosis of diabetes mellitus and intermediate hyperglycemia. WHO, Geneva, pp 1-3 